
de variables psicológicas, económicas y sociales para identificar factores ocultos de los emprendedores en Barranquilla. Económicas CUC, 37(1), 177-202. DOI: http://dx.doi.org/10.17981/econcuc.37.1.2016.08

\title{
Medición de variables psicológicas, económicas y sociales para identificar factores ocultos de los emprendedores en Barranquilla
}

DOI: http://dx.doi.org/10.17981/econcuc.37.1.2016.08

\author{
Gustavo R. Henríquez Fuentes ${ }^{1}$ \\ Jesus Alvaro Rada Llanos ${ }^{2}$ \\ Alvaro Torrenegra ${ }^{3}$
}

\begin{abstract}
Resumen
El desarrollo del artículo permite determinar las variables que caracterizan y afectan a los emprendedores de pequeños negocios en la ciudad de Barranquilla, al momento de desarrollar una idea de negocio, formal o informal. A través del uso de métodos cuantitativos y técnicas de estadística inferencial, se identifican que las variables ocultas impulsan el emprendimiento como parte de la solución al desarrollo sostenible de Barranquilla. Para los efectos, se contrastan la teoría de Gnyawali y Fogel (1994) sobre las condiciones para un ambiente emprendedor. Los resultados permiten complementar con el aporte de otros autores, la identificación de las variables desde la academia, con las cuales se desarrolla el instrumento que servirá para medir las opiniones y características de los emprendedores.
\end{abstract}

Palabras clave: emprendimiento; variables de emprendimiento; pequeñas empresas; desarrollo sostenible; Barranquilla-Colombia.

Recibido: 25.2.2016 Devuelto para revisión: 31.3.2016 Aceptado: 28.4.2016

\begin{abstract}
${ }^{1}$ Becario del doctorado en Administración. Universidad del Norte- Barranquilla, Colombia. Magister en Administración de empresa e innovación. Ingeniero de Mercado Publicidad y ventas. Universidad Simón Bolívar-Barranquilla, Colombia. Docente Universidad de la Costa (CUC)- Barranquilla, Colombia. Adscrito al grupo de investigación Innovar del Caribe. Universidad del Norte - Barranquilla, Colombia. Autor de correspondencia, ghenriqu4@cuc.edu.co .

${ }^{2}$ Doctorando en Administración Universidad del Norte-Barranquilla, Colombia. Master Business Administration. Profesor tiempo completo adscrito al grupo de investigación GEMS de la Universidad Autónoma del Caribe- Barranquilla, Colombia. jrada@uac.edu.co

${ }^{3}$ Doctorando en Administración Universidad del Norte-Barranquilla, Colombia. Magister en Administración de empresas. Universidad EAFIT-Colombia. Especialista en Negocios Internacionales. Administrador de empresas. Universidad del NorteBarranquilla, Colombia. Subdirector Centro de Comercio y Servicio (SENA)-Regional Atlántico. atorrenegrab@hotmail.com .
\end{abstract}

The author; licensee Universidad de la Costa - CUC. 


\title{
Measurement of Psychological, Economic and Social Variables to Identify Hidden Factors of Entrepreneurs in Barranquilla
}

\begin{abstract}
The development of the article allows determining the variables that characterize and affect the entrepreneurs of small businesses in the city of Barranquilla, at the moment of developing a business idea, formal or informal. Through the use of quantitative methods and techniques of inferential statistics, it is identified that the hidden variables drive entrepreneurship as part of the solution to the sustainable development of Barranquilla. For the purpose, the theory of Gnyawali and Fogel (1994) on the conditions for an enterprising environment is contrasted. The results allow complementing with the contribution of other authors, the identification of the variables from the academy, with which the instrument is developed that will serve to measure the opinions and characteristics of the entrepreneurs.
\end{abstract}

Keywords: entrepreneurship; Entrepreneurship variables; small companies; sustainable development; Barranquilla, Colombia.

\section{Introducción}

El emprendimiento ha sido abordado desde distintas teorías económicas y sociales con el ánimo de observar su aporte al desarrollo de los países y generación de empresas. El emprendimiento en América Latina mejora la competiti- vidad de las naciones y la productividad de las empresas, al tiempo que reduce los índices de desempleo y mejora los indicadores de creación de empresas. Impulsado por emprendedores, este proceso se mueve gracias a la actitud, actividades $\mathrm{y}$ aspiraciones de personas que creen en sus capacidades y se aventuran a desa- 
rrollar negocios, superando la frustración de los resultados a corto tiempo; además, los emprendedores aprenden sobre el manejo eficiente de recursos e imprimen innovación a sus productos y servicios (Sparano, 2014).

El siguiente artículo presenta un primer apartado donde se aborda la situación del emprendimiento en Barranquilla a partir de la introducción de elementos relacionados con los grupos de pequeños comerciantes con necesidades básicas distintas. El segundo apartado, denominado marco teórico, está basado en la teoría de Gnyawali y Fogel (1994) para su comprensión, con el ánimo de contrastar el estudio contra esta teoría a partir de técnicas estadísticas. El tercer apartado trata de la importancia que revisten las redes sociales para el emprendimiento, profundizando para ello en la exposición de Granovetter (1983). El cuarto apartado explica el aproximamiento metodológico a las variables estudiadas. El apartado cinco presenta, a partir del análisis estadístico basado en análisis factorial, los hallazgos y resultados de la investigación. Finalmente, en el apartado seis se plantean las conclusiones relacionadas con estos hallazgos a partir de la investigación realizada.

\section{El emprendimiento de pequeños comerciantes en Barranquilla}

El emprendimiento puede ser visto como una acción con un objetivo mercantil que se realiza para tener ingresos a partir del intercambio de un bien o servicio. Shane y Venkataraman agregan que "las oportunidades de emprendimiento son aquellas situaciones en las que nuevos bienes, servicios, materias primas y métodos de organización, pueden ser vendidos e introducidos a un precio mayor que sus costos de producción" (como se cita en Guzmán y Trujillo, 2008). La investigación se centrará en aquellas oportunidades o actividades económicas destinadas a superar una dificultad social, o para cubrir las necesidades primarias de las personas. De acuerdo con Bargsted (2013), el emprendimiento social implica elaborar, sustentar y ejecutar actividades para superar una dificultad social y generar un logro para un bien en común de un grupo humano a partir de actividades empresariales o social comunitarias.

Sobre las ventajas que el emprendimiento genera para la economía de los países, Varela (2001) afirma que si un país incrementa el número de empresas 
y logra que éstas crezcan y se mantengan, bajo un proceso continuo, se incrementarán las oportunidades de empleo y bienestar para la comunidad.

Realizando una observación por las calles de Barranquilla (imágenes 1 y 2 ), se pueden identificar emprendimientos de tipo social llevados a cabo por personas con intereses distintos, entre los que sobresalen los económicos y sociales, generados comúnmente por la falta de oportunidades laborales; también se encuentran otros generados por el relacionamiento social del individuo y sus relaciones comerciales.

Los emprendedores en la ciudad, a diario deben sortear muchos obstáculos para aprovechar una oportunidad: lo financiero, la falta de experiencia o relaciones sociales (redes) y la ausencia de políticas de Estado y otros factores que dificultan la generación de empresas y la puesta en marcha de una oportunidad de negocio.

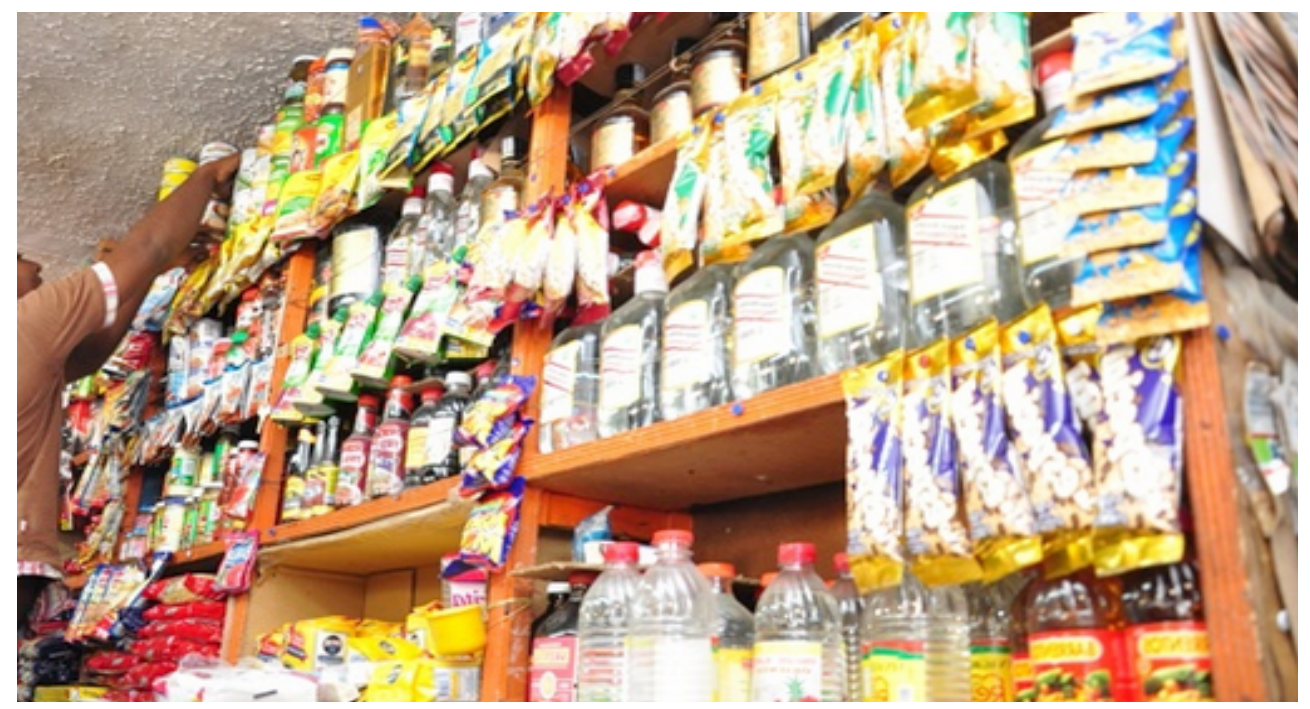

Imagen 1. Tiendas en Barranquilla.

Fuente: adaptado de El Heraldo (2014). 


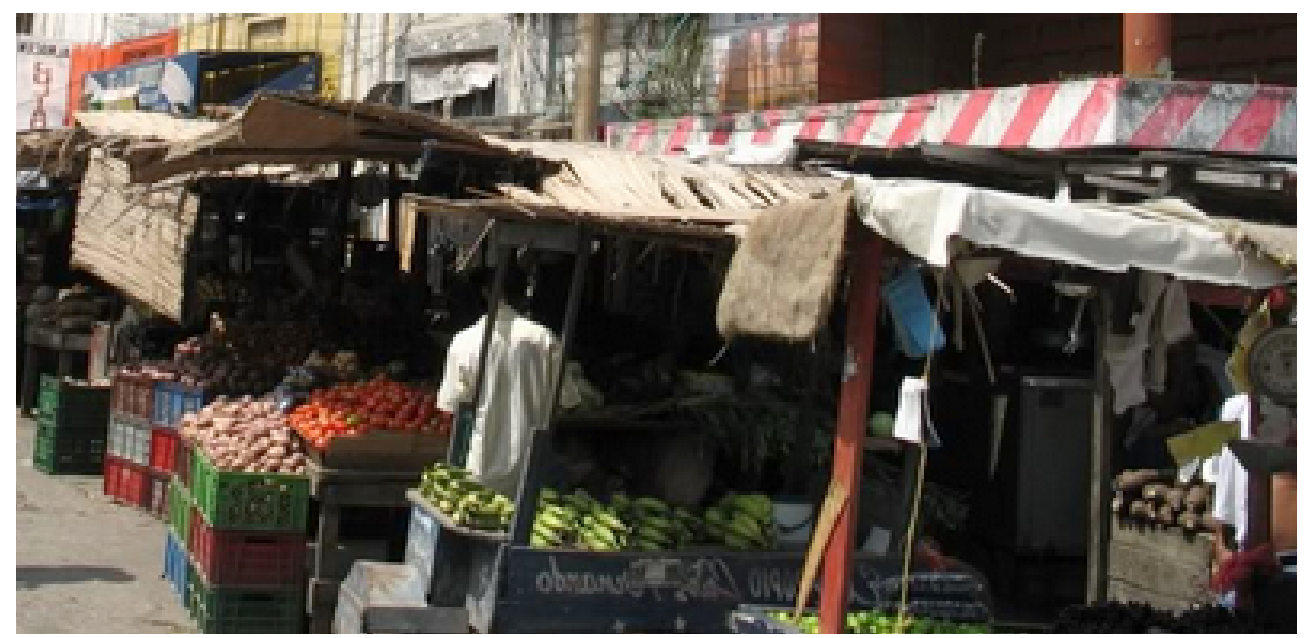

Imagen 2. Comerciantes del centro de Barranquilla.

Fuente: adaptado de Contreras (s.f.)

Con relación a lo anterior, Herrera (2009) dice que un emprendedor, para el desarrollo de sus iniciativas, debe sortear múltiples obstáculos, entre los más difíciles está contar con información y capital financiero y el respaldo simbólico que brinda la legitimación social de su actuación.

Personas de escasos recursos emprenden negocios motivados por la supervivencia, necesitan generar sus ingresos y se enfrentan a diario con obstáculos que son sorteados con el fin de generar ganancias, en ocasiones para sobrevivir, en otras con el ánimo de crecer; como dice la señora
Rosa $^{4}$ quién tiene un pequeño negocio que desarrolló para sobrevivir. Doña Rosa lo define así: "vendo para la papa, usted sabe que el que no trabaja no come... trabajo como para los alimentos y pagar los servicios" (imagen 3).

\footnotetext{
${ }^{4} \mathrm{Su}$ emprendimiento consiste en la venta de aguacates, bananas y bollos, así como de algunos otros productos que, de acuerdo con ella, compra en el mercado público de la ciudad a diario para venderlos en el norte de la ciudad. A diario viaja desde su casa en Santodomingo de Guzmán hasta el norte de la ciudad donde atiende su negocio. Se ha instalado en un espacio pequeño de un conjunto residencial, en el cual lleva, en sus propias palabras, 16 años. Su negocio consiste en una mesa de madera, un banco y un inventario de frutas, que en ocasiones le es "fiado" gracias a las relaciones comerciales con vendedores de frutas y verduras, quienes, según ella lo expresa, "me tratan bien" (consigue descuentos y crédito). Con ellos lleva entre 7 y 8 años de relaciones comerciales.
} 


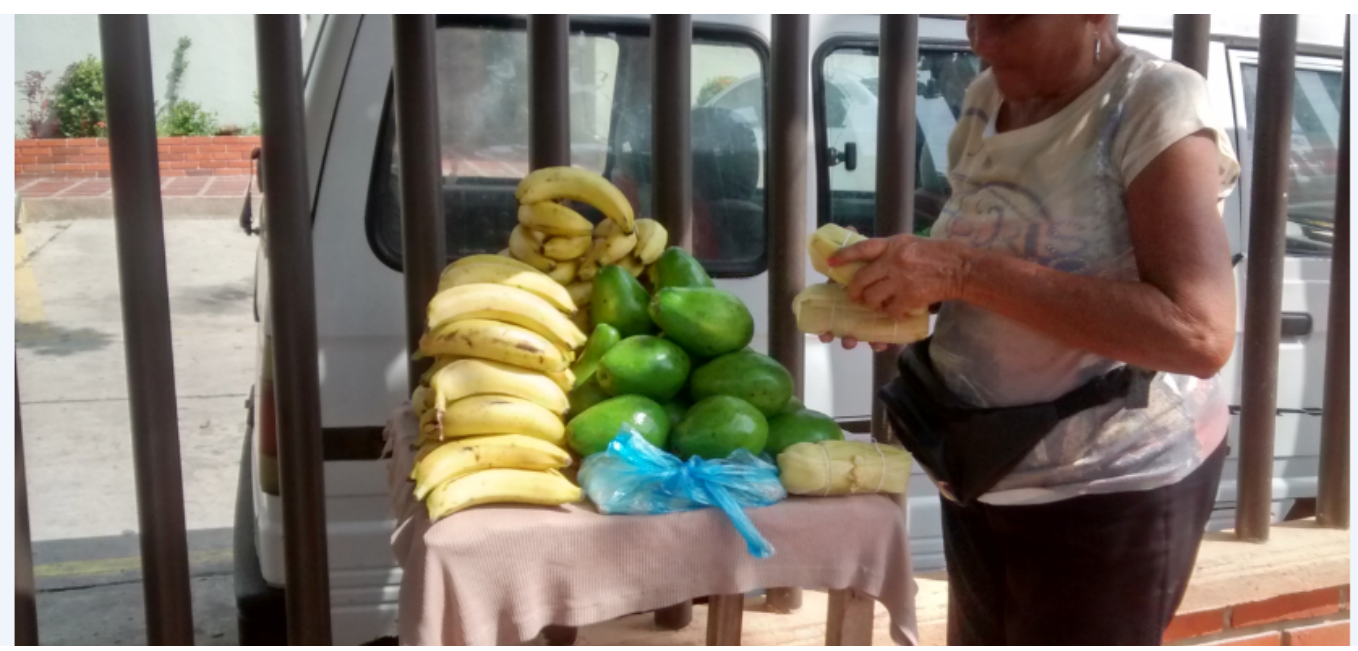

Imagen 3. Comerciante del norte de Barranquilla.

Fuente: Foto tomada por los autores.

Al momento de emprender, una persona necesita amistades o conexiones sociales (redes) que le permitan moverse para conseguir facilidades de crédito, mercancías en consignación o apoyo para la distribución de los productos, ya sea desde lo formal o por medio del mercado informal de bienes y servicios. Sobre la importancia de las redes sociales, Varela (2001) afirma que la única forma como el empresario logra su independencia y el éxito a largo plazo es con el apoyo de una buena red de personas que le brindan soporte cuando lo necesita.

En relación con las consideraciones anteriores, Mokry expresa que "las fuerzas fundamentales que desarrollan el espíritu empresarial, como la familia, la presencia y la eficacia de las redes sociales, o las actitudes de apoyo de la iniciativa empresarial, reciben prácticamente ninguna atención seria de programas de desarrollo económico formal" " (como se cita en Gnyawali y Fogel, 1994).

Las redes sociales son una importante fuente para encontrar conocimientos y habilidades que permiten fortalecer las experiencias propias; además, con ellas se puede obtener financiación, proveedores o distri-

${ }^{5}$ Traducido por el autor 
buidores en la localidad, o el acercamiento, a través de una relación de amistad, a una persona dispuesta a financiar una idea de negocio o comprar parte de la producción.

En cuanto al apoyo de redes sociales o grupos de soporte, Uribola, Escamilla, Fernández y León (2012) sostienen, por ejemplo, que a través de grupos presbiterianos en Querétaro, México se promueve el trabajo y la solidaridad en y con el grupo, haciendo énfasis en emprendimiento y liderazgo.

Las redes sociales generan el dinamismo que contribuye al desarrollo de actividades en grupo. El dinamismo que generan las relaciones entre personas se le puede conocer como capital social, que en palabras de Adler y Kwon son los bienes disponibles a los individuos o a los grupos basados en la estructura y contenido de las relaciones sociales de los actores, con efectos en la información, en la influencia del poder y en la solidaridad que se hace disponible a estos actores sociales (como se cita en Barón y Gómez, 2012).

A través de Gnyawali y Fogel (1994) y su teoría sobre las condiciones para un ambiente emprendedor se desarrollará esta investigación de corte cuantitativo sobre las variables identificadas en la revisión de lectura afín con el pensamiento de estos autores.

\section{Marco teórico}

El emprendimiento puede ser visto como un arte o habilidad que lleva a la creación de empresas o al desarrollo de negocios de diferentes tamaños, estén formalmente constituidos o no. En este sentido Sparano afirma que "el emprendimiento ha sido considerado una de las variables claves del desarrollo, y debe convertirse además en un sello para los nuevos profesionales" (2014, p. 97). De acuerdo con Toca, "el emprendimiento ha sido vinculado con riqueza, empresa, cambio, empleo, valor [y] crecimiento" (2010, p. $45)$.

La creación de empresas y el emprendimiento es una fuente importante de generación de riqueza y empleo para las economías. Urbano y Díaz aseguran que la actividad emprendedora ha obtenido cada día más apoyo de las administraciones públicas, y gracias a ellas (instituciones) se ha fomentado más la actividad del emprendimiento, apoyado de la academia y su interés en esta actividad (2009). Las pequeñas y medianas empresas (pymes) son importantes en sus localidades y comunidades, ya que suministran bienes y servicios adaptados a la comunidad, con costo asequible a los locales; además, son una fuente importante de empleo, especialmente para trabajadores poco calificados como mujeres y jóvenes ( $\mathrm{SNV}$ y WBCSD, s.f.) 
Toca (2010) asegura que el emprendimiento tiene un componente importante para el desarrollo económico de una región, por ello reviste de un interés y asociatividad entre diferentes partes involucradas para lograr su multiplicación a través de la enseñanza. Asimismo, esta autora dice sobre los emprendedores que "deben aprender a generar sus propias respuestas frente a los acontecimientos del entorno y dejar de buscarlas en experiencias pasadas, tienen que aprender más sobre el saber que sobre el hacer" (2010, p. 52). Toca hace un gran énfasis en la capacitación que debe tener un emprendedor para enfrentar situaciones que pueden garantizar el desarrollo de su negocio, aun por encima del entrenamiento y la experiencia que deba tener. La autora deja ver la importancia de la enseñanza sobre emprendimiento pues les permite a los emprendedores generar competencias que los lleven a hacer frente a problemas diversos.

De la mano con Toca, y bajo la línea de los autores que piensan en la necesidad de enseñar sobre emprendimiento, se encuentran Correa, Delgado y Conde (2011) quienes dicen que la enseñanza del emprendimiento en las universidades debe estar basada en el desarrollo de habilidades, atributos, competencias y maneras de pensar y comportarse en diferentes situaciones.
Estos autores diseñan una tabla ${ }^{6}$ de competencias emprendedoras (Tabla 1) de la cual sobresalen, entre las primeras ranqueadas, los riesgos calculados, la iniciativa/autonomía y la innovación y creatividad. Correa et al. recalcan la importancia de actitudes y competencias para el arte del emprendedor. Las competencias pueden ser impartidas en centros de educación superior para cultivar el arte del emprendimiento en los estudiantes.

En este sentido Gutiérrez, Asprilla y Gutiérrez orientan sus esfuerzos de investigación hacia el emprendimiento en universidades hacia la investigación en innovación (2014). Para los autores, las empresas viven lo que ellos llaman "momentos creativos e investigativos" y desarrollar un fuerte dominio de competencias gerenciales ${ }^{7}$ les permitirá alcanzar objetivos de productividad y competitividad.

\footnotetext{
${ }^{6}$ Para efectos de concentración en las principales, sólo se toman tres de las competencias. Correa, Delgado y Conde (2011, p. 47), disponible en http://www.scielo.org.co/pdf/ ean/n71/n71a04.pdf

Pensamiento estratégico, táctico operativo; mejorar, innovar y optimizar los procesos de la organización; identificar y optimizar los procesos de negocio de la organización; elaborar, evaluar y administrar proyectos empresariales; detectar oportunidades para emprender nuevos negocios y/o desarrollar nuevos productos.
} 
Tabla 1

Competencias emprendedoras de uso frecuente en literatura especializada

\begin{tabular}{llcc}
\hline No. & \multicolumn{1}{c}{ Categoría- Competencia emprendedora } & Frecuencia & Porcentaje \\
\hline 1 & Riesgos calculados & 26 & 70.3 \\
2 & Iniciativa/autonomía & 22 & 59.5 \\
3 & Innovación/creatividad & 21 & 56.8 \\
4 & Orientación a resultados & 17 & 45.9 \\
5 & Confianza en él mismo & 13 & 35.1 \\
6 & Desarrollo de relaciones & 13 & 35.1 \\
7 & Perseverancia & 12 & 32.4 \\
8 & Pensamiento estratégico & 11 & 29.7 \\
9 & Búsqueda de información & 10 & 27 \\
10 & Capacidad de planificación y organización & 10 & 27 \\
11 & Compromiso/productividad & 9 & 24.3 \\
12 & Dinamismo/energía & 9 & 24.3 \\
13 & Responsabilidad & 9 & 24.3 \\
14 & Calidad del trabajo & 8 & 21.6 \\
15 & Impacto e influencia & 8 & 21.6 \\
16 & Control directivo & 7 & 18.9 \\
17 & Liderazgo & 7 & 18.9 \\
18 & Locus control interno & 7 & 18.9 \\
19 & Resolución de problemas & 7 & 18.9 \\
20 & Integridad & 6 & 16.2 \\
\hline
\end{tabular}

Fuente: tomado de Correa, Delgado y Conde (2011).

El emprendedor necesita conocer el entorno, o rodearse de personas que lo conozcan para disminuir su posibilidad de fracaso. También puede desarrollar estudios o contratar asesorías que le permitan conocer las condiciones del entorno, su política o regu- lación para empresas, normatividad y carga impositiva, entre muchos factores. Para ello necesita unas reglas de juego claras que le permitan moverse rápidamente generando negocio, o aliviando su carga de impuestos para estimularlos. 
Concentrando los esfuerzos en Barranquilla, se encuentran estudios, por ejemplo, como el de Orozco y Arraut (2014), donde a través de la influencia de las instituciones se estima el peso de las políticas y de las instituciones en general de tipo formal e informal en la competitividad de Barranquilla, Cartagena, Santa Marta y Sincelejo. De acuerdo con Orozco y Arraut (2014), en el enfoque institucional, el empresario se condiciona por factores formales e informales del entorno, implementando normativas y reglamentaciones concernientes con la actividad emprendedora a partir de su propio aprendizaje y experiencia y de un proceso de socialización, en el cual debe tener en cuenta organizaciones políticas, económicas, sociales y educativas.

Bajo la óptica de las instituciones, de acuerdo con Orozco y Arraut (2014), los emprendedores encuentran apoyo al fomento de una cultura empresarial y de innovación; a sus actitudes como emprendedores; al desarrollo de habilidades, educación y entrenamiento; a la transferencia de investigación y desarrollo, el desarrollo de negocios por iniciativas no financieras; así como políticas de apoyo financiero.

También deben tener en cuenta las costumbres de la sociedad que permiten una mejor actividad emprendedora o la entorpecen. Según North (1991), las instituciones son limitaciones para la actividad humana, sean éstas formales o informales; entre las instituciones formales se encuentran reglas, normas y leyes, todas escritas para ser respetadas. Entre las informales están, entre otras, las costumbres, creencias, convenciones, es decir, códigos no escritos que complementan las instituciones formales.

En este aspecto, los autores concluyen que, en la región, la percepción de políticas que estimulan la actividad emprendedora es muy baja, lo que indica que el gobierno poco o nada apoya esta actividad.

En conclusión, se puede indicar que existen variables de distintos tipos que permiten el desarrollo del emprendimiento, desde las psicológicas, como la asunción del riesgo y la actitud para emprender; las de enfoque económico como las instituciones; o las que van de la mano con el desarrollo de factores sociales, como el desempleo, relaciones sociales, la difusión de la enseñanza en emprendimiento para adquirir habilidades, entre otras.

El estudio se desarrolla tomando como base el constructo de Gnyawali y Fogel (1994) con elementos que permiten el desarrollo de nuevas oportunidades de negocio; entre estos elementos están los aspectos psicológicos, sociales o económicos que influyen en la creación de empresas, además, se complementa con las teorías de autores varios sobre el ambiente propicio para el 


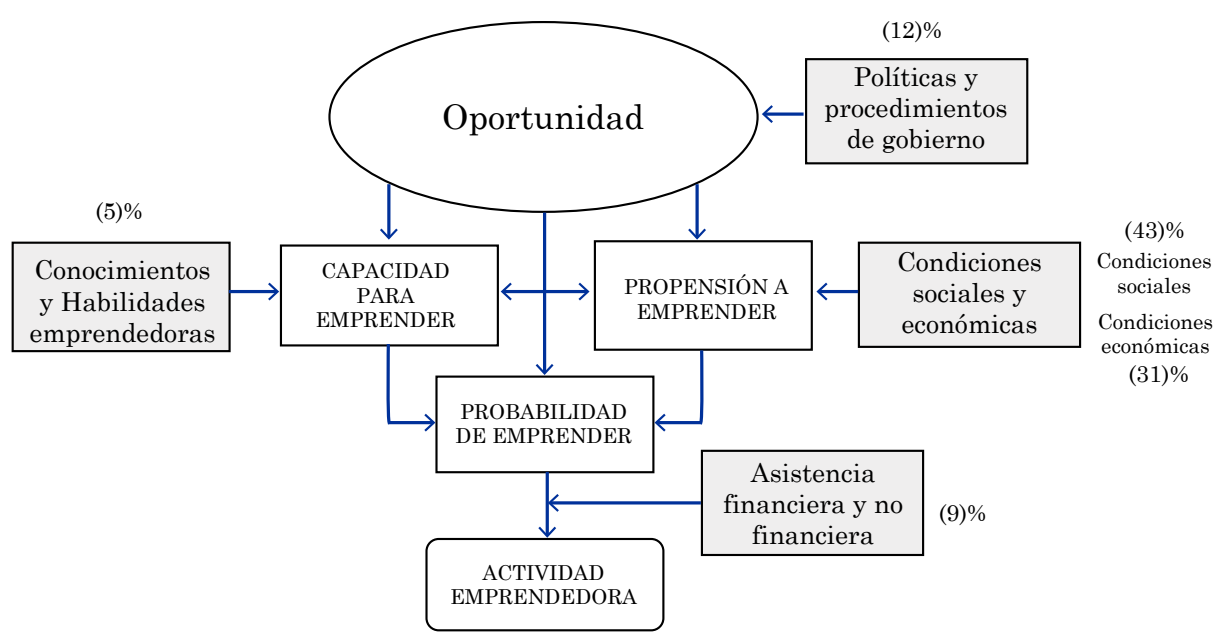

Figura 1. Modelo de Gnyawali y Fogel y artículos basados en el GEM. Fuente: Álvarez y Urbano adaptado de Gnyawali y Fogel, 1994 (2011).

emprendimiento. Gnyawali y Fogel (1994) hablan de los elementos principales para crear empresas bajo un modelo integrativo estimulante del ambiente emprendedor (Figura 1).

Sobre el modelo de Gnyawali y Fogel, Álvarez y Urbano (2011) identifican, a través de una revisión de la literatura en el Global Entrepreneurship Monitor (GEM), las áreas de oportunidad para investigar sobre los elementos principales enumerados por Gnyawali y Fogel.

Álvarez y Urbano describen el modelo como la manera en que los emprendedores a través de variables de tipo psicológicas, sociales y económicas intentan hacerse a una oportunidad del mercado. Para ello deben sortear, en primera medida, las regulaciones y políticas gubernamentales, además de los procedimientos que pueden entorpecer la generación de empresas. Una vez superada las regulaciones y políticas, intervienen en el proceso la capacidad y la propensión de los emprendedores, es decir, conocimientos y habilidades emprendedoras y condiciones sociales y económicas, respectivamente. La suma de las dos aumenta o disminuye la probabilidad de emprender. Esta probabilidad se ve fa- 
vorecida o disminuida en la medida en que existan programas o leyes de fomento al emprendimiento de asistencia financiera y no financiera por parte del gobierno. Es de esta manera como se construye un ambiente propicio para la actividad emprendedora.

Las oportunidades de negocio las proporciona el mercado en la medida en que la oferta por productos y servicios no está satisfecha en su totalidad. De acuerdo con Sparano (2014), en países desarrollados, la oportunidad de emprendimiento baja a medida que los sectores se hacen fuertes, lo que a su vez crea fuentes de empleo, contrario a lo que sucede en países no desarrollados (Colombia) donde los sectores no alcanzan a desarrollarse completamente, dejando vacíos u oportunidades de negocios a personas; en este caso, por el contrario, se llevan a cabo muchos emprendimientos por necesidad.

Bargsted habla del emprendimiento (para su caso emprendimiento social) con beneficios para la sociedad facilitado por experiencias previas de fracaso y de éxito y alimentado por intereses y aspiraciones laborales (2013). Arnold, señala que "al mismo tiempo, estas experiencias dependen de las oportunidades educativas y sociales a las que nos vemos expuestos" (como se cita en Bargsted, 2013, p. 128).

\section{La importancia de las redes sociales en} el emprendimiento

Otro punto importante para el desarrollo de emprendimiento son las conexiones del emprendedor con personas de diversas profesiones y sectores, lo cual le beneficia en el desarrollo de su tarea emprendedora. Aunque Gnyawali y Fogel, no profundizan mucho en el tema de las redes sociales, se abordarán éstas por la relevancia que representan para el éxito de un emprendimiento.

En su teoría hablan de las redes sociales como uno de los elementos a tener en cuenta que generan condiciones para un ambiente emprendedor. Es Granovetter (1983) quien en una revisión de la teoría de las redes sociales a la que denomina la fuerza de los lazos débiles, afirma que éstas apoyan a las personas en la búsqueda de afinidades de tipo social, tipo económico, desarrollo de negocios o para afianzar vínculos buscando asociación para el bien común.

De acuerdo con Granovetter, a través de un lazo débil de amistad con alguien se puede obtener un beneficio, gracias al lazo fuerte de amistad de este conocido con otro individuo al cual el emprendedor podrá recurrir con fines comerciales o de otro tipo.

El argumento de Granovetter (1983) afirma que nuestros conocidos (lazos débiles) tienen menos probabilidades de estar invo- 
lucrados socialmente entre sí que nuestros amigos (lazos fuertes), con quienes existen lazos estrechos; es así como el individuo y sus conocidos conforman una red de baja densidad (una en la que muchas de las posibles líneas de relación están ausentes), mientras que el conjunto que consiste en el mismo individuo y sus amigos más cercanos estará densamente entretejido (muchas de las posibles líneas están presentes), reforzando los lazos débiles o estrechos y socializando a las personas a través de estos.

Butler y Hansen, sobre redes sociales para emprendimiento, reconocen tres etapas: la fase de emprendimiento, el start-up y la fase de funcionamiento (1991). Butler y Hansen, tal como se explica en la figura 2, manifiestan que en la fase de emprendimiento anterior al start-up, la red social del emprendedor es extremadamente importante, porque le asegura el acceso a fuentes de recursos de diversa índole.

Durante la fase del start-up, las redes se transforman en redes de negocios al enfocarse en establecer conexiones con individuos y organizaciones que pueden servir a las necesidades inmediatas de la nueva empresa (como se cita en Herrera, 2009, p. 25).

Herrera, 2009; Phelan et al. 2006, afirman que un "emprendedor que participa de una red más desarrollada (en términos del

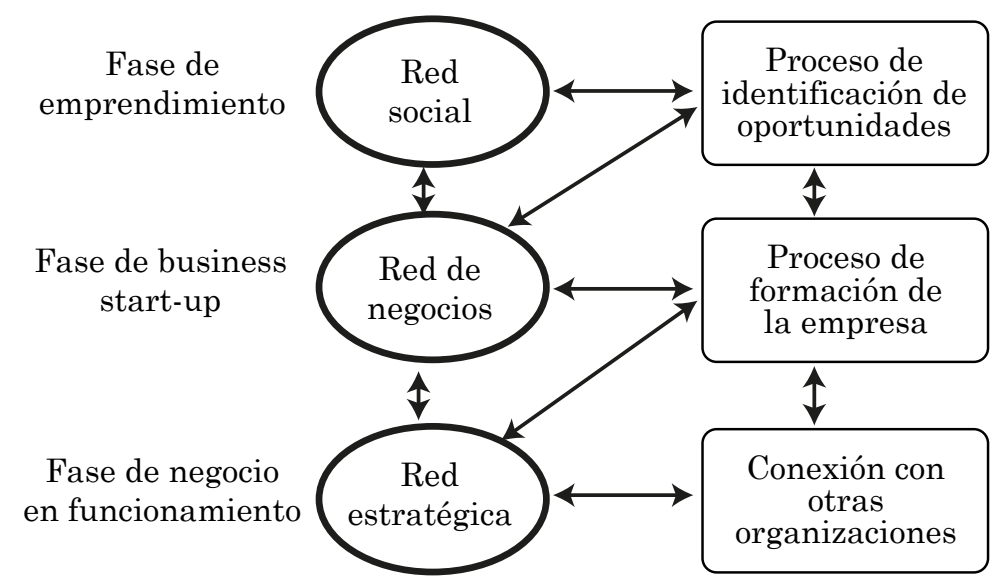

Figura 2. Modelo de Butler y Hansen.

Fuente: tomado de Herrera, 2009. 
número y la calidad de los lazos creados), tendrá mayor posibilidad de acceder a recursos escasos, incrementando la probabilidad de éxito del emprendimiento" (como se cita en González y Zúñiga, 2011, p. 82).

De acuerdo con la revisión del estado del arte realizada, se diseñó la Tabla 2 para resumir las teorías que se ajustan o complementan el aporte de Gnyawali y Fogel (1994). En ésta se relacionan los autores, su principal aporte a esta investigación, la re- lación de su aporte con la teoría de Gnyawali y Fogel y las variables a las que apuntan sobre el emprendimiento (para el caso, éstas serán llamadas en adelante macrovariables y darán origen al diseño del instrumento). Se desea lograr un análisis a partir de las coincidencias de estas teorías y sus elementos en común para estudiar el fenómeno desde el punto de vista cuantitativo, basando los resultados en la certeza y la confianza que se puede tener en las matemáticas y la estadística para el estudio de las variables.

Tabla 2

Revisión del estado del arte que complementa la teoría de las claves para un ambiente emprendedor de Gnyawali y Fogel.

\begin{tabular}{|c|c|c|c|c|}
\hline Autor & $\begin{array}{l}\text { Aporte a los objetivos de la } \\
\text { investigación }\end{array}$ & $\begin{array}{c}\text { Elemento } \\
\text { relacionado } \\
\text { con Gnyawali } \\
\text { y Fogel para } \\
\text { emprender } \\
\text { aventura }\end{array}$ & $\begin{array}{c}\text { Macro- } \\
\text { variables }\end{array}$ & Convención \\
\hline $\begin{array}{l}\text { Sparano } \\
(2014)\end{array}$ & $\begin{array}{l}\text { Emprendimiento por necesidad y poco } \\
\text { desarrollo de sectores genera oportunidad } \\
\text { en países no desarrollados }\end{array}$ & $\begin{array}{l}\text { Propensión a } \\
\text { emprender }\end{array}$ & Desempleo & DESE \\
\hline $\begin{array}{l}\text { Sparano } \\
(2014)\end{array}$ & $\begin{array}{l}\text { Propensión a emprender debido a } \\
\text { condiciones sociales y económicas }\end{array}$ & $\begin{array}{l}\text { Propensión a } \\
\text { emprender }\end{array}$ & $\begin{array}{l}\text { Necesidad } \\
\text { económica } \\
\text { y social }\end{array}$ & NEES \\
\hline $\begin{array}{l}\text { Arnold } \\
\text { (2001, } \\
\text { (citado por } \\
\text { Bargsted, } \\
\text { 2013). }\end{array}$ & $\begin{array}{c}\text { El emprendimiento es facilitado por } \\
\text { experiencias de éxito y fracaso previas al } \\
\text { desarrollo de la oportunidad, que a su vez } \\
\text { dependen de oportunidades educativas } \\
\text { y sociales de las personas }\end{array}$ & $\begin{array}{l}\text { Capacidad } \\
\text { para } \\
\text { emprender }\end{array}$ & $\begin{array}{l}\text { Nivel de } \\
\text { educación }\end{array}$ & NEDU \\
\hline
\end{tabular}




\begin{tabular}{|c|c|c|c|c|}
\hline \multirow[b]{2}{*}{$\begin{array}{l}\text { Herrera } \\
(2009)\end{array}$} & \multirow[b]{2}{*}{$\begin{array}{l}\text { Cómo las redes sociales facilitan la } \\
\text { puesta en marcha de emprendimientos }\end{array}$} & \multirow[b]{2}{*}{$\begin{array}{l}\text { Condiciones } \\
\text { sociales / } \\
\text { conocimientos }\end{array}$} & \multirow[b]{2}{*}{$\begin{array}{l}\text { Relaciones sociales } \\
\text { Experiencia } \\
\text { compartida }\end{array}$} & \multirow[b]{2}{*}{$\begin{array}{l}\text { RESO } \\
\text { EXCO }\end{array}$} \\
\hline & & & & \\
\hline $\begin{array}{l}\text { Toca } \\
(2010)\end{array}$ & $\begin{array}{l}\text { Importancia de la enseñanza del } \\
\text { emprendimiento y la multidisciplinariedad }\end{array}$ & $\begin{array}{l}\text { Capacidad para } \\
\text { emprender }\end{array}$ & $\begin{array}{l}\text { Enseñanza del } \\
\text { emprendimiento }\end{array}$ & ENEM \\
\hline Correa & Enseñanza del emprendimiento & Capacidad para & Nivel de educación & NEDU \\
\hline$(2011)$ & enfatizando el desarrollo de competencias & emprender & Competencias & COMP \\
\hline $\begin{array}{l}\text { Orozco y } \\
\text { Arraut } \\
(2014)\end{array}$ & $\begin{array}{l}\text { El empresario está condicionado a un marco } \\
\text { de leyes en donde implementa, gracias a su } \\
\text { aprendizaje, socialización y experiencia, su } \\
\text { oportunidad de emprendimiento. }\end{array}$ & $\begin{array}{l}\text { Políticas y } \\
\text { procedimientos } \\
\text { de Gobierno }\end{array}$ & $\begin{array}{l}\text { Regulaciones } \\
\text { del Gobierno }\end{array}$ & REGU \\
\hline $\begin{array}{l}\text { Granovetter } \\
\quad(1983)\end{array}$ & $\begin{array}{l}\text { La importancia de los vínculos de amistad y } \\
\text { relaciones sociales para construir relaciones } \\
\text { de bien común entre desconocidos y } \\
\text { miembros de una colectividad }\end{array}$ & $\begin{array}{l}\text { Condiciones } \\
\text { sociales / } \\
\text { conocimientos }\end{array}$ & $\begin{array}{l}\text { Relaciones } \\
\text { sociales }\end{array}$ & RESO \\
\hline \multirow{3}{*}{$\begin{array}{l}\text { Borges, } \\
\text { Salete, } \\
\text { Camargo } \\
\text { y Borges } \\
(2013)\end{array}$} & \multirow{4}{*}{$\begin{array}{l}\text { Aprendizaje empresarial a } \\
\text { través de emprendimientos } \\
\text { sustentables por medio de tres } \\
\quad \text { tipos de aprendizaje: } \\
\text { ación personal y social, aprendizaje por } \\
\text { experiencias del contexto, aprendizaje } \\
\text { la negociación con emprendimientos }\end{array}$} & \multirow{3}{*}{$\begin{array}{l}\text { Capacidad } \\
\text { para } \\
\text { emprender }\end{array}$} & $\begin{array}{l}\text { Experiencia } \\
\text { compartida }\end{array}$ & EXCO \\
\hline & & & $\begin{array}{l}\text { Mejoras en } \\
\text { procesos / } \\
\text { Interactuar con } \\
\text { clientes }\end{array}$ & INTC \\
\hline & & & Nivel de educación & NEDU \\
\hline \multirow{3}{*}{$\begin{array}{l}\text { Mora } \\
(2011)\end{array}$} & & \multirow{3}{*}{$\begin{array}{l}\text { Propensión } \\
\text { a } \\
\text { emprender }\end{array}$} & Asunción de riesgo & ASRI \\
\hline & \multirow[t]{2}{*}{$\begin{array}{l}\text { Las actitudes emprendedoras, sensibilidad } \\
\text { a desarrollar empresa. El rol de las } \\
\text { creencias para asumir riesgos medidos }\end{array}$} & & $\begin{array}{l}\text { Factores y } \\
\text { actitudes } \\
\text { psicológicos }\end{array}$ & FACS \\
\hline & & & Creencias & CREE \\
\hline $\begin{array}{l}\text { Crissien } \\
\text { (2011). }\end{array}$ & $\begin{array}{l}\text { Desarrollo de programas en la } \\
\text { universidad para enseñar a las } \\
\text { personas sobre emprendimiento y } \\
\text { ser empresarios, ayudando al } \\
\text { desarrollo económico de la región. }\end{array}$ & $\begin{array}{l}\text { Capacidad } \\
\text { para } \\
\text { emprender }\end{array}$ & Nivel de educación & NEDU \\
\hline
\end{tabular}

Fuente: autores basado en otros autores. 


\section{Metodología}

La investigación tiene por objetivo medir las variables psicológicas, económicas y sociales para identificar los factores ocultos que motivan a los emprendedores en Barranquilla, desde la teoría de Gnyawali y Fogel sobre las dimensiones clave que generan un ambiente emprendedor. En otras palabras, se desea entender las características que poseen los emprendedores que generan la creación de pequeñas empresas a partir de la teoría de Gnyawali y Fogel (1994); también se desea descubrir los factores ocultos del emprendimiento en esta ciudad y de los cuales se apoyan los emprendedores para aprovechar oportunidades del mercado.

El método de estudio. La investigación es de tipo deductivo basado en la lógica de la investigación cuantitativa bajo la teoría de Gnyawali y Fogel (1994). La investigación se apoyó en un trabajo de campo bajo las bondades de una encuesta en escala, haciendo la verificación de esta teoría bajo la interpretación de los datos empíricos que arroje el estudio. (Bonilla, Hurtado y Jaramillo, 2009).

Se pretende utilizar técnicas estadísticas de interdependencia, como el análisis factorial exploratorio (AFE) basado en componentes principales. Según Díaz, Díaz, González y Henao, las técnicas de interdependencia son conocidas como "técnicas con un carácter eminentemente descriptivo que permiten un mejor conocimiento de la realidad medida por estas variables" (2013, p. 7).

Tal como lo expresan Frías-Navarro y Pascual:

La contribución del AFE al avance del conocimiento científico es muy valiosa. Facilitar el análisis de los patrones de interrelación entre las variables, reducir datos, clasificarlos y describirlos son cuestiones que el AFE resuelve de forma eficiente en todas las áreas de las ciencias sociales (2012, p. 46).

Aspectos metodológicos y caracterización de la muestra. Será utilizado el muestreo no probabilístico de fácil estudio, cuyo método consiste en evaluar con base en la percepción de un conjunto de clientes a quienes es fácil acceder (Serna, 2006).

Para el caso, se desarrollará la encuesta en pequeños comerciantes o emprendedores, formales o no formales cuyas empresas sean unipersonales o no sobrepasen un tamaño de 10 empleados.

La investigación tiene como objetivo medir las variables psicológicas, económicas y sociales a través de una encuesta tipo Likert para descubrir los factores ocultos entre emprendedores en Barranquilla que permiten la generación de nuevas empresas o de casos de emprendimiento informales; además, realiza un análisis de correlación para descubrir las relaciones matemáticas directas entre las 
variables identificadas. Todo lo anterior basado en la teoría de Gnyawali y Fogel (1994).

Se diseñó la tabla 3 con el propósito de presentar de una manera ordenada las variables que hacen parte del estudio y su convención para facilitar la interpretación y el análisis de datos.

Tabla 3

Variables y convención de las variables.

\begin{tabular}{clc}
\hline & Variables & Convenciones \\
\hline 1 & Desempleo & DESE \\
2 & $\begin{array}{l}\text { Factores sociales y situación } \\
\text { económica }\end{array}$ & NEES \\
3 & Creencias y costumbres & CREE \\
4 & Nivel de educación alto & NEDU \\
5 & Actitud & FACS \\
6 & Relaciones personales & RESO \\
7 & $\begin{array}{l}\text { Experiencias y enseñanzas } \\
\text { compartidas }\end{array}$ & EXCO \\
8 & $\begin{array}{l}\text { Enseñanza sobre } \\
\text { emprendimiento }\end{array}$ & ENEM \\
9 & $\begin{array}{l}\text { Desarrollo de competencias } \\
10\end{array}$ & $\begin{array}{l}\text { Regulaciones } \\
\text { gubernamentales }\end{array}$ \\
\multirow{2}{*}{11} & $\begin{array}{l}\text { Interactuar con clientes / } \\
\text { Aprendizaje empresarial }\end{array}$ \\
\hline
\end{tabular}

Fuente: autores.

\section{Hallazgos y resultados de la investigación}

Mediante el test de Barlett (figura 3) se logra demostrar que "la matriz de coeficientes de correlación poblacional no es significativamente distinta de la matriz de identidad" (Díaz, Díaz, González y Henao, 2013, p. 50); con esto se afirma que el estudio se puede realizar por métodos cuantitativos, en este caso la teoría afirma que si el estimado (335) es mayor que el tabulado $(38,9)$, se demuestra que hay dependencia entre las variables.

En la tabla 4 se resume, con datos obtenidos a través del programa SPSS, el nivel de significancia chi cuadrada y el índice de Kaiser-Meyer-Olkin.

Tabla 4

KMO y prueba de Barlett

\begin{tabular}{lll}
\hline \multicolumn{2}{c}{$\begin{array}{c}\text { Medida de adecuación muestral } \\
\text { de Kaiser-Meyer-Olkin. }\end{array}$} & \multicolumn{1}{c}{, 768} \\
\hline Prueba de & Chi-cuadrado aproximado & 149,667 \\
esfericidad & Gl & 55 \\
de Bartlett & Sig. &, 000 \\
\hline
\end{tabular}

Fuente: datos obtenidos del programa SPSS.

$$
\begin{aligned}
& -a \log n V>X^{2}, \text { donde } a=N-1-(2 P+5) / 6 \quad y \quad V=|R| \\
& {[-48,5][-6,90]>X_{0,05, \frac{11(11-1)}{2}}^{2}=335,02>38,95 \rightarrow \text { estimado }>\text { tabulado }}
\end{aligned}
$$

Figura 3. Test de Barlett (prueba de independencia de variables). 
El resultado 0,768, por encima de 0,5 en el índice KMO, indica que es viable trabajar con los métodos cuantitativos, ya que además se tiene un nivel de significancia muy cercano a cero (0). Se corrieron las 11 variables con las que se ha trabajado para obtener la tabla 5 de la varianza total explicada en el programa SPSS bajo la técnica de análisis factorial o de reducción de factores.
En esta tabla se observa que la concentración del $57 \%$ de la variabilidad de los datos está en los tres componentes con autovalores mayores a 1; sin embargo, por tratarse de 11 variables, y para no perder la mitad de la variabilidad, se prueba trabajando la técnica de extracción con los 5 primeros componentes ya que los componentes 4 y 5 tienen autovalores muy cercanos a 1 , con 0,951 para el caso del cuarto componente y 0,877 para el quinto componente.

Tabla 5

Varianza total explicada por 3 factores.

Método de extracción componentes principales.

\begin{tabular}{|c|c|c|c|c|c|c|}
\hline \multirow{2}{*}{ Componente } & \multicolumn{3}{|c|}{ Autovalores iniciales } & \multicolumn{3}{|c|}{$\begin{array}{c}\text { Sumas de las saturaciones al cuadrado } \\
\text { de la extracción }\end{array}$} \\
\hline & Total & $\begin{array}{c}\% \text { de la } \\
\text { varianza }\end{array}$ & $\begin{array}{c}\% \\
\text { acumulado }\end{array}$ & Total & $\begin{array}{c}\% \text { de la } \\
\text { varianza }\end{array}$ & $\%$ acumulado \\
\hline 1 & 3,731 & 33,923 & 33,923 & 3,731 & 33,923 & 33,923 \\
\hline 2 & 1,358 & 12,342 & 46,264 & 1,358 & 12,342 & 46,264 \\
\hline 3 & 1,186 & 10,783 & 57,048 & 1,186 & 10,783 & 57,048 \\
\hline 4 &, 951 & 8,644 & 65,692 & & & \\
\hline 5 &, 877 & 7,971 & 73,663 & & & \\
\hline 6 &, 717 & 6,520 & 80,183 & & & \\
\hline 7 &, 599 & 5,449 & 85,632 & & & \\
\hline 8 &, 542 & 4,924 & 90,556 & & & \\
\hline 9 & ,418 & 3,798 & 94,354 & & & \\
\hline 10 &, 362 & 3,294 & 97,648 & & & \\
\hline 11 & ,259 & 2,352 & 100,000 & & & \\
\hline
\end{tabular}

Fuente: autores a través del programa SPSS. 
La matriz de componentes de esta corrida indica que no es factible trabajar con 5 componentes ya que lo correcto es asignar al menos dos variables para cada componente. En esta oportunidad se observa que el componente 1 obtiene 7 variables, mientras los demás componentes obtienen solamente una variable, configurándose como componentes triviales (Tabla 6).

Tabla 6

Matriz de componentes con extracción de 5 factores. Método de extracción componentes principales.

\begin{tabular}{lccccc}
\hline \multirow{2}{*}{ Convención } & \multicolumn{5}{c}{ Componente } \\
\cline { 2 - 6 } & $\mathbf{1}$ & $\mathbf{2}$ & $\mathbf{3}$ & $\mathbf{4}$ & $\mathbf{5}$ \\
\hline ESE & $\mathbf{, 6 1 5}$ &, 319 &,- 109 &, 136 &,- 074 \\
NEES & $\mathbf{, 5 5 4}$ &, 181 &, 466 &,- 143 &,- 269 \\
CREE &, 357 & $\mathbf{, 5 5 5}$ &, 342 &, 330 &,- 262 \\
NEDU &, 277 &, 604 &, 102 &,- 209 & $\mathbf{, 6 6 4}$ \\
FACS &, 237 &,- 434 & $\mathbf{, 6 6 8}$ &, 377 &, 206 \\
RESO & $\mathbf{, 6 9 9}$ &, 107 &,- 097 &,- 042 &,- 330 \\
EXCO & $\mathbf{, 5 7 4}$ &,- 516 &, 298 &,- 317 &, 078 \\
ENEM & $\mathbf{, 8 1 8}$ &,- 019 &,- 052 &,- 083 &, 291 \\
COMP & $\mathbf{, 7 4 8}$ &,- 158 &,- 218 &,- 021 &,- 018 \\
REGU &, 444 &,- 167 &,- 369 & $\mathbf{, 6 7 9}$ &, 196 \\
INTEC & $\mathbf{, 7 3 6}$ &,- 178 &,- 317 &,- 218 &,- 095 \\
\hline
\end{tabular}

Fuente: autores a través del programa SPSS.

Se corrió en el programa SPSS con extracción de $3,4,5$ y 6 componentes y en todos los casos los resultados fueron parecidos: el componente uno obtiene entre
7 y 8 variables, mientras los demás sólo uno.

En extracción de 3, el componente 1 obtiene 7 , el componente 2 obtiene 2 y el 3 obtiene 1 variable, por ello se decide trabajar con extracción de 2 componentes y una variabilidad del 46\%; despreciando el componente 3 que, aunque obtiene un autovalor por encima de $1(1,186)$, termina convirtiéndose, en la extracción de 3 , en componente trivial.

La matriz del componente queda como lo muestra la tabla 7 :

Tabla 7

Matriz de componentes extracción 2 factores.

Método de extracción componentes principales.

\begin{tabular}{lcc}
\hline \multirow{2}{*}{ Convención } & \multicolumn{2}{c}{ Componente } \\
\cline { 2 - 3 } & $\mathbf{1}$ & $\mathbf{2}$ \\
\hline DESE & $\mathbf{, 6 1 5}$ &, 319 \\
NEES & $\mathbf{, 5 5 4}$ &, 181 \\
CREE &, 357 & $\mathbf{, 5 5 5}$ \\
NEDU &, 277 & $\mathbf{, 6 0 4}$ \\
FACS &, 237 & $\mathbf{- , 4 3 4}$ \\
RESO & $\mathbf{6 9 9}$ &, 107 \\
EXCO & $\mathbf{, 5 7 4}$ &,- 516 \\
ENEM & $\mathbf{, 8 1 8}$ &,- 019 \\
COMP & $\mathbf{, 7 4 8}$ &,- 158 \\
REGU & $\mathbf{4 4 4}$ &,- 167 \\
INTC & $\mathbf{7 3 6}$ &,- 178 \\
\hline
\end{tabular}

Fuente: autores a través del programa SPSS. 


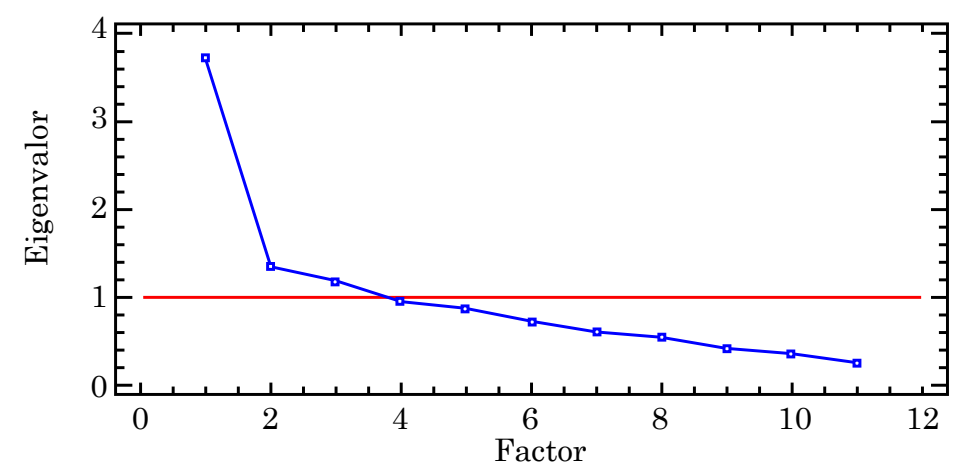

Gráfica 1. Gráfica de sedimentación de las variables.

Fuente: autores basados en el programa Statgraphics.

La gráfica de sedimentación de los componentes sugiere hacer corte a partir de 3 variables, sin embargo, por los motivos ya explicados, se decide identificar solamente dos factores ocultos o latentes dentro de las características que llevan a emprender en Barranquilla. La gráfica 1 muestra la gráfica de sedimentación obtenida a través de Statgraphics.

Esta gráfica sugiere que el corte de los eigenvalores (autovalores) se debe realizar en los tres componentes primeros, con una distancia muy corta con los componentes 4 , 5 y 6 , así como distancia muy mínima entre componente 2 y $3(10,78 \%)$ en variabilidad.

De esta forma, el componente 1 se ha denominado: labor de universidad, gobierno y redes sociales y el componente 2 se ha llamado: factor psicológico y educación formal.
La investigación arrojó múltiples tipos de negocios o empresas, desde aquellas establecidas con 20 empleados, otras de 1 o 2 empleados con Cámara de Comercio y Registro Único Tributario y todos los papeles en regla, hasta otros negocios bajo la informalidad total. Dentro de la muestra no existe un tipo de negocio que destaque entre los demás en cantidad, sin embargo, si es bueno analizar su diferencia en cuanto a la actividad (tabla 8).

La muestra es bastante heterogénea, sólo sobresale el negocio de venta de minutos y no por mucha ventaja, poniendo en evidencia que las personas en Barranquilla tienden a llenar esos vacíos que dejan las empresas en muchos sectores, sobre todo en la venta de productos y servicios de bajo costo como aguacate, frutas, panes, minutos, víveres y productos para el hogar. 
Tabla 8

Caracterización de la muestra.

\begin{tabular}{lclc}
\hline Tipo de Negocio & Total & & Total \\
\hline Distribución de alumbrado público y elementos eléctricos & 1 & Venta de frutas & 3 \\
Cancha sintética de futbol & 1 & Sai (venta de minutos celular) & 4 \\
Floristería & 1 & Taxista & 1 \\
Productos para el hogar & 1 & Venta de jugos & 2 \\
Banquetes y distribuidor de teléfonos & 1 & Comerciante & 2 \\
Restaurante & 2 & Transcripciones & 2 \\
Educación - jardín infantil & 2 & Dueño de finca & 1 \\
Venta y mantenimiento de relojes & 1 & Venta de helado & 1 \\
Venta de libros & 1 & Venta de chorizos & 1 \\
Venta de gafas & 1 & Panadería & 1 \\
Venta de dulces y pasabocas & 3 & Venta de aguacates & 2 \\
Diseñadora de moda & 1 & Venta de comida a domicilio & 1 \\
Venta de bicicleta y mantenimiento & 1 & Tienda & 3 \\
Vendedor de pescado & 1 & Papelería - droguería & 1 \\
Diseño gráfico & 1 & Peluquería & 1 \\
Miscelánea y variedades & 1 & Farmacia & 1 \\
Cuidado y lavado de carros & 2 & Internet y llamadas & 2 \\
Accesorios celulares & 2 & Proveedores materiales eléctricos & 1 \\
\hline
\end{tabular}

Fuente: autores.

\section{Conclusiones}

A través del estudio realizado se puede afirmar que no son pocos los emprendedores que demuestran interés por la educación y su aporte al emprendimiento, a pesar que en la mayoría de casos no poseen un nivel alto, escasamente, bachillerato. 


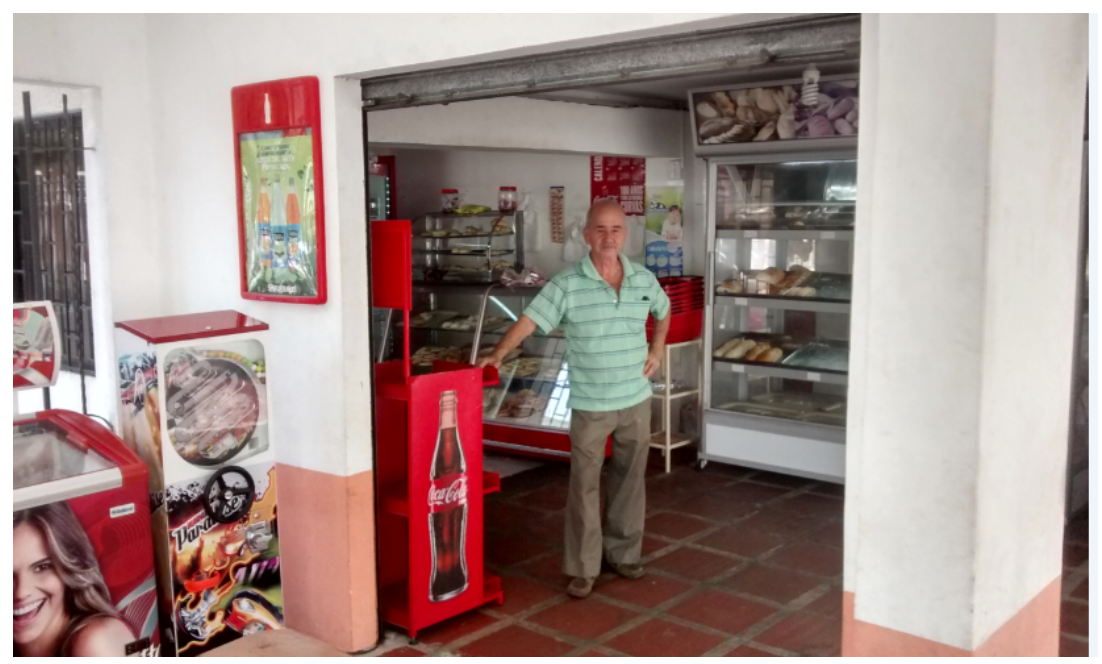

Imagen 4. Comerciante del norte de Barranquilla. Panadería y helados.

Fuente: Fotografía de los autores.

El factor uno recoge características propias del entorno y de la labor de la universidad y el Gobierno para apoyar al emprendimiento, así como el aprendizaje y provecho que se obtiene de otros emprendedores y de relaciones sociales; por ello se ha denominado labor de universidad, gobierno y redes sociales.

Por otro lado, se encuentra el factor dos, el cual se identifica más con características personales del emprendedor, ya sea que las posea o desee alcanzarlas, como su afectación con las creencias y costumbres, su actitud de cara al emprendimiento y el nivel de educación; este componente se ha llamado factor psicológico y educación formal.

Las condiciones de muchos de ellos eran difíciles, reflejadas en la manera de vestir o por el lugar de su residencia, en algunos casos suburbios o barrios subnormales de Barranquilla. En la mayoría de los casos, las personas abordadas fueron muy amables en el desarrollo de la investigación, lo cual demuestra afinación de sus habilidades comerciantes. Otros, aunque no demostraban un alto grado de amabilidad, tampoco demostraron lo contrario y permitieron su trato. 
Hubo algunas complicaciones en el desarrollo de la investigación como personas que no permitieron ser encuestadas por considerarlo una pérdida de tiempo o por que se encontraban a su modo de expresarlo "muy ocupadas para perder el tiempo".

Se encuestaron algunos emprendedores con una infraestructura organizada, que consiste en un pequeño local, como el caso de las tiendas, panaderías, floristerías, algunos negocios de venta de minutos a celular e Internet, educación y jardín infantil. Otros tipos de negocio de emprendimiento obedecen más a una estructura de organización muy simple, consistente en el inventario de mercancía (en ocasiones en consignación o fiado) y una mesa, carretilla, silla, o simplemente, una toalla en la cual colocan sobre el suelo sus mercancías para la venta, por ejemplo ventas de aguacates, frutas, libros y chorizos. Otros emprendedores, como el vendedor de pescado o el que se dedica al cuidado y lavado de carros, sólo cargan consigo un balde en el cual almacenan de manera un poco precaria el pescado envuelto en papel cartón y, para el caso del cuidador y lavador de carros, para almacenar el agua con la que lava los autos.
Los anteriores tipos de emprendimiento se encuentran en los dos factores identificados.

\section{Investigaciones futuras}

Se recomienda la realización de una encuesta en otro grupo de emprendedores con estas características para tener mayor cantidad de datos y proceder a una clasificación estadística a través de la técnica de conglomerados. También se recomienda la realización de una entrevista y la realización de una observación para proceder a un análisis comparativo entre las categorías de emprendedores de tipo social que se identifiquen y los grupos o clústeres de emprendedores identificados bajo el análisis de tipo cuantitativo.

\section{Referencias}

Álvarez, C., y Urbano, D. (2011). Una década de investigación basada en el GEM: Logros y retos. Academia Revista Latinoamericana de Administración, (46): 16-37.

Bargsted, M. (2013). El emprendimiento social desde una mirada psicosocial. Civilizar, 13(25): 121-132. 
Barón, L. y Gómez, R. (2012). Relaciones sociales y sensación de conexidad. Usos de cibercafés, telecentros y bibliotecas en Colombia. Anuario Electrónico de Estudios en Comunicación Social “Disertaciones”, 5(1): 105-128.

Bonilla, E., Hurtado, J. y Jaramillo, C. (2009). La investigación. Aproximaciones a la construcción del conocimiento científico. Bogotá: Alfaomega.

Borges, J., Salete, M., Camargo, A. y Borges, C. (2013). El aprendizaje empresarial en los emprendimientos sustentables del sector turístico del cerrado brasileño. Estudios y Perspectivas en Turismo, 22(3): 568-582.

Butler, J. y Hansen, G. (1991). Network evolution, entrepreneurial success, and regional development. Entrepreneurship and Regional Development, 3(1): 1-16.

Contreras, (s.f). [Fotografía]. Recuperado de http://www.skyscrapercity.com/ forumdisplay.php?f=3548

Correa, Z., Delgado, C. y Conde, Y. (2011). Formación en emprendimiento en estudiantes de la carrera de administración de empresas en la universidad pública de Popayán. Escuela de Administración de Negocios, (71): 40-51.
Crissien, J. (2011). Enseñando entrepreneurship creación de empresas. Estrategias pedagógicas para despertar el espíritu empresarial. Escuela de Administración de Negocios, (71): 100-125.

Díaz, M., Díaz, M.E., González, A. y Henao, A. (2013). Introducción al análisis estadístico multivariado aplicado. Barranquilla: Universidad del Norte.

El Heraldo. (2014). El Heraldo.com [Fotografía]. Recuperado de http://www. elheraldo.co/economia/tiendas-de-barrio-son-los-negocios-mas-numerososen-barranquilla-146661

Frías-Navarro, D. y Pascual, M. (2012). Prácticas del análisis factorial exploratorio (AFE) en la investigación sobre conducta del consumidor y marketing. Suma Psicológica. 19(1): 47-58.

Gnyawali, D. y Fogel, D. (1994). Enviroments for entrepreneurship development: Key Dimensions and research implications. Entrepreneurship Theory and Practice (ET\&P), 43-62.

González, R. y Zúñiga, A. (2011). Método CEPCES para la evaluación del potencial emprendedor. Journal of Technology Management \& Innovation, 6(1). 77-99. doi: http://dx.doi.org/10.4067/ S0718-27242011000100008 
Granovetter, M. (1983). The strength of weak ties: a network theory revisited. Sociological Theory (1): 201-233.

Gutiérrez, J. A., Asprilla, E. y Gutiérrez, J.M. (2014). Emprendimiento e investigación en la escala de la formación profesional y la innovación empresarial en Colombia. Escuela de Administración de Negocios, (76): 144-157.

Guzmán, A. y Trujillo, M. (2008). Emprendimiento social-Revisión de literatura. Estudios Gerenciales, 24(109): 105-125.

Herrera, H. (2009). Investigación sobre redes sociales y emprendimiento: revisión de la literatura y agenda futura. Innovar, 19(33): 19-33.

Mora, R. (2011). Estudio de actitudes emprendedoras con profesionales que crearon empresa. Escuela de Administración de Negocios. (71): 70-83.

North, D. (1991). Institutions. Journal of economic perspective, 5(1): 97-112.

Orozco, J. y Arraut, L. (2014). El entorno competitivo para el emprendimiento en la región Caribe de Colombia: caso de Barranquilla, Cartagena, Santa Marta y Sincelejo. Escuela de Administración de Negocios, (74): 86-105.
Serna, H. (2006). Servicio al cliente una nueva visión: clientes para siempre, Metodología y herramientas para medir la lealtad y satisfacción. Bogotá: 3R.

Servicio Holandés de Cooperación al Desarrollo (SNV) y Consejo Empresarial Mundial para el Desarrollo Sostenible (WBCSD). (s.f). Guía empresarial para los actores del desarrollo 2004. Recuperado de http:// www.wbcsd.org/web/publications/sldevactors.pdf

Sparano, H. (2014). Emprendimiento en América Latina y su impacto en la gestión de proyectos. Dimensión Empresarial, 12(2): 95-106.

Toca, C. (2010). Consideraciones para la formación en emprendimiento: explorando nuevos ámbitos $\mathrm{y}$ posibilidades. Estudios Gerenciales, 26(117): 41-60.

Urbano, D. y Díaz, J. (2009). Creación de empresas e instituciones. En R. Hernández, M. Fuentes y L. Rodríguez (Ed.), Creación de empresas: aproximación al estado del arte. (95-109). Lisboa: Juruá. 
Uribola, A., Escamilla, C., Fernández., M. y León, E. (2012). Emprendedurismo, ética calvinista y desarrollo regional. Anuario Electrónico de Estudios en Comunicación Social "Disertaciones", 5(2): 80-102.
Varela, R. (2001). Innovación empresarial. Arte y ciencia en la creación de empresas. Bogotá: Prentice Hall. 